\title{
Growth order of Meromorphic Solutions of Higher-order Linear Differential Equations
}

JunfEng Xu

Department of Mathematics, Shandong University, Jinan, Shandong, 250100, P. R. China.

e-mail : xujunf@gmail.com

ZhANLIANG ZhANG

Department of Mathematics, Zhaoqing University, Zhaoqing, Guangdong, 526061, P. R. China.

e-mail : zlzhang@zqu.edu.cn

ABstract. In this paper, we investigate higher-order linear differential equations with entire coefficients of iterated order. We improve and extend the result of L. Z. Yang by using the estimates for the logarithmic derivative of a transcendental meromorphic function due to Gundersen and the extended Wiman-Valiron theory by Wang and Yi. We also consider the nonhomogeneous linear differential equations.

\section{Introduction and main results}

In this paper, we shall assume that the reader is familiar with the fundamental results and the standard notation of the Nevanlinna value distribution theory of meromorphic functions (see [12], [8]). The term "meromorphic function" will mean meromorphic in the whole complex plane $\mathbb{C}$.

The linear measure of a set $E \subset[0,+\infty)$ is defined as $m(E)=\int_{0}^{+\infty} \chi_{E}(t) d t$. The logarithmic measure of a set $E \subset[1,+\infty)$ is defined by $\operatorname{lm}(E)=\int_{1}^{+\infty} \chi_{E}(t) / t d t$, where $\chi_{E}(t)$ is the characteristic function of $E$. The upper and lower densities of $E$ are

$$
\overline{\operatorname{dens}} E=\limsup _{r \rightarrow+\infty} \frac{m(E \cap[0, r])}{r}, \quad \underline{\operatorname{dens}} E=\liminf _{r \rightarrow+\infty} \frac{m(E \cap[0, r])}{r} .
$$

For $k \geq 2$, we consider a linear differential equation

$$
A_{k} f^{(k)}+A_{k-1} f^{(k-1)}+\cdots+A_{0} f=0,
$$

Received November 30, 2006.

2000 Mathematics Subject Classification: 34M10, 30D35.

Key words and phrases: meromorphic function, growth order, Wiman-Valiron theory.

Supported by Specialized Research Fund for the Doctoral Program of Higher Education (20060422049) and NSF of China (10771121). 
where $A_{0}, \cdots, A_{k}$ are entire functions with $A_{0} \not \equiv 0$. If $A_{k} \equiv 1$, it is well known that all solutions of (1.1) are entire functions, and if some of the coefficients of (1.1) are transcendental, then (1.1) has at least one solution with order $\sigma(f)=\infty$.

Thus the question which arises is: What conditions on $A_{0}, \ldots, A_{k-1}$ will guarantee that every solution $f \not \equiv 0$ of $(1.1)$ has infinite order if $A_{k} \equiv 1$ ?

For the above question, there are many results for second order linear differential equations (see for example [5],[7],[4],[2]).

In 2005, L.Z. Yang considered the higher order linear differential equations and obtained the following result.

Theorem 1.1([13, Theorem 1]). Let $H$ be a set of complex numbers satisfying $\overline{\operatorname{dens}}\{|z|: z \in H\}>0$, and let $A_{0}(z), A_{1}(z), \cdots, A_{k}(z)$ be entire functions and satisfy

$$
\begin{gathered}
\left|A_{0}(z)\right| \geq e^{\alpha|z|^{\mu}}, \\
\left|A_{j}(z)\right| \leq e^{\beta|z|^{\mu}}, \quad j=1, \ldots, k,
\end{gathered}
$$

as $z \rightarrow \infty$ for $z \in H$. Then every meromorphic (or entire) solution $f \not \equiv 0$ of (1.1) satisfies $\sigma(f)=\infty$ and $\sigma_{2}(f) \geq \mu$.

Theorem 1.2([13, Theorem 2]). Let $H$ be a set of complex numbers satisfying $\overline{\operatorname{dens}}\{|z|: z \in H\}>0$, and let $A_{0}(z), A_{1}(z), \cdots, A_{k-1}(z)$ be entire functions and $A_{k}(z) \equiv 1$ such that

$$
\max \left\{\sigma\left(A_{j}\right): j=1, \cdots, k-1\right\} \leq \sigma\left(A_{0}\right)=\sigma<+\infty
$$

and for some constants $0 \leq \beta<\alpha$ and for any $\varepsilon>0$ sufficiently small, we have

$$
\begin{gathered}
\left|A_{0}(z)\right| \geq e^{\alpha|z|^{\sigma-\varepsilon}}, \\
\left|A_{j}(z)\right| \leq e^{\beta|z|^{\sigma-\varepsilon}}, \quad j=1, \cdots, k,
\end{gathered}
$$

as $z \rightarrow \infty$ for $z \in H$. Then every solution $f \not \equiv 0$ of (1.1) satisfies $\sigma(f)=\infty$ and $\sigma_{2}(f)=\sigma\left(A_{0}\right)$.

Remark 1. In Theorem 1.2 , we note $A_{k}(z) \equiv 1$, this is to say, all the solutions of equation (1.1) are entire functions. There will be a question to rise: If $A_{k}(z)$ is entire, what can be stated about the solutions of equation (1.1)? Obviously, the solution $f(z)$ of the equation (1.1) can be meromorphic function. In general, the Wiman-Valiron theory will only be in effect for the solution of entire function $f(z)$. In this paper, we avoid the difficulty by using the method of Wang and Yi and obtain the following result:

Theorem 1.3. Let $H$ be a set of complex numbers satisfying $\overline{\operatorname{dens}}\{|z|: z \in H\}>0$, and let $A_{0}(z), A_{1}(z), \ldots, A_{k-1}(z), A_{k}(z)$ be entire functions such that

$$
\max \left\{\sigma\left(A_{j}\right): j=1, \cdots, k\right\} \leq \sigma\left(A_{0}\right)=\sigma<+\infty ;
$$


and for some constants $0 \leq \beta<\alpha$ and for any $\varepsilon>0$ sufficiently small, we have

$$
\begin{gathered}
\left|A_{0}(z)\right| \geq e^{\alpha|z|^{\sigma-\varepsilon}}, \\
\left|A_{j}(z)\right| \leq e^{\beta|z|^{\sigma-\varepsilon}}, \quad j=1, \cdots, k,
\end{gathered}
$$

as $z \rightarrow \infty$ for $z \in H$. Then every meromorphic solution $f \not \equiv 0$ of (1.1) satisfies $\sigma(f)=\infty$ and $\sigma_{2}(f)=\sigma\left(A_{0}\right)$.

Now there exists another question: For so many solutions of infinite order, how to describe precisely the properties of growth of solutions of infinite order of (1.1)?

In this paper, we improve and extend Theorem 1.1 and Theorem 1.3 by making use of the concept of iterated order. For $r \in[0, \infty)$, we define $\exp _{1} r=e^{r}$ and $\exp _{i+1} r=\exp \left(\exp _{i} r\right)(i \in \mathbb{N})$. For $r$ sufficiently large, we define $\log _{1} r=\log r$, $\log _{i+1} r=\log \left(\log _{i} r\right)(i \in \mathbb{N})$. To express the rate of growth of entire function of infinite order, we introduce the notion of iterated order (see [9], [1]).

Definition 1. The iterated $i$-order of a meromorphic function $f$ is defined by

$$
\sigma_{p}(f)=\limsup _{r \rightarrow \infty} \frac{\log _{p} T(r, f)}{\log r} \quad(p \in \mathbb{N}) .
$$

Remark 2. (1). If $p=1$, then we denote $\sigma_{1}(f)=\sigma(f)$; (2). If $p=2$, then we denote by $\sigma_{2}(f)$ the so-called hyper order (see [14]).

Definition 2. The finiteness degree of the order of a meromorphic function $f$ is defined by

$$
i(f)= \begin{cases}0 & \text { if } f \text { is rational, } \\ \min \left\{j \in \mathbb{N}: \sigma_{j}(f)<\infty\right\} & \text { if } f \text { is transcendental with } \\ & \sigma_{j}(f)<\infty \text { for some } j \in \mathbb{N}, \\ \infty & \text { if } \sigma_{j}(f)=\infty, \forall j \in \mathbb{N} .\end{cases}
$$

Definition 3. The iterated convergence exponent of the sequence of zeros of a meromorphic function $f$ is defined by

$$
\lambda_{i}(f)=\limsup _{r \rightarrow \infty} \frac{\log _{i} n(r, 1 / f)}{\log r} \quad(i \in \mathbb{N}) .
$$

Theorem 1.4. Let $H$ be a set of complex numbers satisfying $\overline{\operatorname{dens}}\{|z|: z \in H\}>0$, and let $A_{0}(z), A_{1}(z), \cdots, A_{k}(z)$ be entire functions and satisfy

$$
\left|A_{0}(z)\right| \geq \exp _{p}\left\{\alpha|z|^{\mu}\right\},
$$




$$
\left|A_{j}(z)\right| \leq \exp _{p}\left\{\beta|z|^{\mu}\right\}, \quad j=1, \cdots, k-1, k
$$

as $z \rightarrow \infty$ for $z \in H$, where $0 \leq \beta<\alpha, \mu>0,1 \leq p<\infty$. Then every meromorphic (or entire) $f \not \equiv 0$ of (1.1) satisfies $\sigma_{p+1}(f) \geq \mu$.

Theorem 1.5. Let $H$ be a set of complex numbers satisfying $\overline{\operatorname{dens}}\{|z|: z \in$ $H\}>0$, and let $A_{0}(z), A_{1}(z), \cdots, A_{k}(z)$ be entire functions of iterated order with $\max \left\{\sigma_{p}\left(A_{j}\right): j=1, \cdots, k\right\} \leq \sigma_{p}\left(A_{0}\right)=\sigma<+\infty, 1 \leq p<\infty$ such that for some constants $0 \leq \beta<\alpha$ and for any given $\varepsilon>0$, we have

$$
\begin{gathered}
\left|A_{0}(z)\right| \geq \exp _{p}\left\{\alpha|z|^{\sigma-\varepsilon}\right\}, \\
\left|A_{j}(z)\right| \leq \exp _{p}\left\{\beta|z|^{\sigma-\varepsilon}\right\}, \quad j=1, \cdots, k-1, k,
\end{gathered}
$$

as $z \rightarrow \infty$ for $z \in H$. Then every solution $f \not \equiv 0$ of $(1.1)$ satisfies $\sigma_{p+1}(f)=$ $\sigma_{p}\left(A_{0}\right)=\sigma$.

Considering nonhomogeneous linear differential equations

$$
A_{k} f^{(k)}+A_{k-1} f^{(k-1)}+\cdots+A_{0} f=F
$$

corresponding to (1.1), we obtain the following results of the iterated order and iterated convergence exponent of zeros of solutions of (1.1).

Theorem 1.6. Let $F, A_{0}(z), A_{1}(z), \cdots, A_{k}(z)$ satisfy the hypotheses of Theorem 1.5 , and let $F \neq 0$ be an entire function of iterated order with $i(F)=q$.

(i) If $q<p+1$ or $q=p+1, \sigma_{p+1}(F)<\sigma_{p}\left(A_{0}\right)$, then every solution $f(z)$ of $(1.2)$ satisfies $\bar{\lambda}_{p+1}(f)=\lambda_{p+1}(f)=\sigma_{p+1}(f)=\sigma$, with at most one exceptional solution $f_{0}$ satisfying $i\left(f_{0}\right)<p+1$ or $\sigma_{p+1}\left(f_{0}\right)<\sigma$.

(ii) If $q>p+1$ or $q=p+1, \sigma_{p}\left(A_{0}\right)<\sigma_{p+1}(F)<+\infty$, then every solution $f(z)$ of $(1.2)$ satisfies $i(f)=q$ and $\sigma_{q}(f)=\sigma_{q}(F)$.

\section{Lemmas}

Lemma 2.1.([10]) Let $F(r)$ and $G(r)$ be monotone nondecreasing functions on $(0, \infty)$ such that $(i) F(r) \leq G(r)$ n.e. or (ii) for $r \neq H \cup[0,1]$ having finite logarithmic measure, then for any constant $\alpha>1$, there exists $r_{0}>0$ such that $F(r) \leq G(\alpha r)$ for all $r>r_{0}$.

Lemma 2.2(Gundersen [6]). Let $f$ be a transcendental meromorphic function of finite order $\sigma$. Let $\varepsilon>0$ be a constant, and $k$ and $j$ be integers satisfying $k>j \geq 0$. Then the following two statements hold:

(a) There exists a set $E_{1} \subset(1, \infty)$ which has finite logarithmic measure, such that for all $z$ satisfying $|z| \notin E_{1} \bigcup[0,1]$, we have

$$
\left|\frac{f^{(k)}(z)}{f^{(j)}(z)}\right| \leq|z|^{(k-j)(\sigma-1+\varepsilon)} .
$$


(b) There exists a set $E_{2} \subset[0,2 \pi)$ which has linear measure zero, such that if $\theta \in[0,2 \pi)-E_{2}$, then there is a constant $R=R(\theta)>0$ such that (2.3) holds for all $z$ satisfying $\arg z=\theta$ and $R \leq|z|$.

Lemma 2.3 (Gundersen [6]). Let $f$ be a transcendental meromorphic function. Let $\alpha>1$ be a constant, and $k$ and $j$ be integers satisfying $k>j \geq 0$. Then the following two statements hold:

(a) There exists a set $E_{1} \subset(1, \infty)$ which has finite logarithmic measure, and a constant $C>0$, such that for all $z$ satisfying $|z| \notin E_{1} \bigcup[0,1]$, we have (with $r=|z|)$

$$
\left|\frac{f^{(k)}(z)}{f^{(j)}(z)}\right| \leq C\left[\frac{T(\alpha r, f)}{r}(\log r)^{\alpha} \log T(\alpha r, f)\right]^{k-j} .
$$

(b) There exists a set $E_{2} \subset[0,2 \pi)$ which has linear measure zero, such that if $\theta \in[0,2 \pi)-E_{2}$, then there is a constant $R=R(\theta)>0$ such that (2.2) holds for all $z$ satisfying $\arg z=\theta$ and $R \leq|z|$.

Lemma 2.4([3], Lemma 1). Let $g(z)$ be a meormorphic function with $\sigma(g)=\beta<$ $\infty$. Then for any $\varepsilon>0$, there exists a set $E \subset(1, \infty)$ with $\operatorname{lm} E<\infty$, such that for all $z$ with $|z|=r \notin([0,1] \cup E), r \rightarrow \infty$, then

$$
|g(z)| \leq \exp \left\{r^{\beta+\varepsilon}\right\} .
$$

Applying Lemma 2.5 to $1 / g(z)$, it is clearly that for any given $\varepsilon>0$, there exists a set $E \subset(1, \infty)$ with $\operatorname{lm} E<\infty$, such that for all z with $|z|=r \notin([0,1] \cup E), r \rightarrow \infty$, then

$$
\exp \left\{-r^{\beta+\varepsilon}\right\} \leq|g(z)| \leq \exp \left\{r^{\beta+\varepsilon}\right\} .
$$

It is well known that the Wiman-Valiron theory (see[10]) is an indispensable device while considering the growth of entire solution of a complex differential equation. In order to consider the growth of meromorphic function solutions of a complex differential equation, Wang and Yi [11] extended the Wiman-Valiron theory from entire functions to meromorphic functions. Here we give the special form where meromorphic function has infinite order:

Lemma 2.5. Let $f(z)=g(z) / d(z)$ be the infinite order meromorphic function and $\sigma_{2}(f)=\alpha$, where $g(z)$ and $d(z)$ are entire functions, $\sigma(d)<\infty$, there exists a sequence $r_{j}\left(r_{j} \rightarrow \infty\right)$ satisfying $z_{j}=r_{j} e^{i \theta_{j}}, \theta_{j} \in[0,2 \pi), \lim _{j \rightarrow \infty} \theta_{j}=\theta_{0} \in[0,2 \pi)$, $\left|g\left(z_{j}\right)\right|=M\left(r_{j}, g\right)$ and $j$ is sufficient large, we have

$$
\frac{f^{(n)}\left(z_{j}\right)}{f\left(z_{j}\right)}=\left(\frac{\nu_{g}\left(r_{j}\right)}{z_{j}}\right)^{n}(1+o(1))(n \in \mathbb{N}),
$$




$$
\limsup _{r \rightarrow \infty} \frac{\log \log \nu_{g}(r)}{\log r}=\sigma_{2}(g)=\alpha .
$$

Similar with the proof of Lemma 2.5, we can obtain the following result. Here we omit the detail.

Lemma 2.6. Let $f(z)=g(z) / d(z)$ be the infinite order meromorphic function and $\sigma_{p}(f)=\alpha,(p>1)$, where $g(z)$ and $d(z)$ are entire functions, $\sigma_{p-1}(d)<\infty$, there exists a sequence $r_{j}\left(r_{j} \rightarrow \infty\right)$ satisfying $z_{j}=r_{j} e^{i \theta_{j}}, \theta_{j} \in[0,2 \pi), \lim _{j \rightarrow \infty} \theta_{j}=\theta_{0} \in$ $[0,2 \pi),\left|g\left(z_{j}\right)\right|=M\left(r_{j}, g\right)$ and $j$ is sufficient large, we have

$$
\begin{gathered}
\frac{f^{(n)}\left(z_{j}\right)}{f\left(z_{j}\right)}=\left(\frac{\nu_{g}\left(r_{j}\right)}{z_{j}}\right)^{n}(1+o(1))(n \in \mathbb{N}), \\
\limsup _{r \rightarrow \infty} \frac{\log _{p} \nu_{g}(r)}{\log r}=\sigma_{p}(g)=\alpha .
\end{gathered}
$$

Lemma 2.7(Kinnunen [9, Remark 1.3]). If $f$ is a meromorphic function with $i(f)=p \geq 1$, then $\sigma_{p}(f)=\sigma_{p}\left(f^{\prime}\right)$.

Lemma 2.8([1]). Let $f(z)$ be a meromorphic solution of the differential equation

$$
f^{(k)}+B_{k-1} f^{(k-1)}+\cdots+B_{0} f=F,
$$

where $B_{0}, \cdots, B_{k-1}, F \not \equiv 0$ are meromorphic functions, such that

(1) $\max \left\{i(F), i\left(B_{j}\right)(j=0, \cdots, k-1)\right\}<i(f):=p+1,(0<p<\infty)$; or

(2) $\max \left\{\sigma_{p}(F), \sigma_{p}\left(B_{j}\right)(j=0, \cdots, k-1)\right\}<\sigma_{p+1}(f)$.

Then $\bar{\lambda}_{p+1}(f)=\lambda_{p+1}(f)=\sigma_{p+1}(f)$.

\section{Proof of main results}

Proof of Theorem 1.3. Let $f \not \equiv 0$ be a meromorphic solution of (1.1). By using the same arguments as in Theorem 1.1, we have $\sigma(f)=\infty$.

Now for any given $\varepsilon>0$, by the result of Theorem 1.1, we have $\sigma_{2}(f) \geq \sigma-\varepsilon$. Since $\varepsilon$ is arbitrary, we get $\sigma_{2}(f) \geq \sigma\left(A_{0}\right)$.

On the other hand, we can rewrite (1.1) to

$$
1=-\left(\frac{A_{k}}{A_{0}} \frac{f^{(k)}}{f}+\frac{A_{k-1}}{A_{0}} \frac{f^{(k-1)}}{f}+\cdots+\frac{A_{1}}{A_{0}} \frac{f^{\prime}}{f}\right) .
$$

Obviously, the poles of $f$ must be the poles and zeros of $A_{j}(j=0,1, \cdots, k-1, k)$, then $\lambda(1 / f)<\sigma<\infty$. By Hadmard factorization theorem, we know $f$ can write to $f(z)=\frac{g(z)}{d(z)}$, where $g(z)$ and $d(z)$ are entire functions, and $\lambda(d)=\sigma(d)=$ 
$\lambda(1 / f) \leq \sigma<\infty, \sigma_{2}(f)=\sigma_{2}(g)$. By Lemma 2.5, for any small $\varepsilon>0$, there exists a sequence $r_{k}\left(r_{k} \rightarrow \infty\right)$ satisfying $z_{k}=r_{k} e^{i \theta_{k}}, \theta_{k} \in[0,2 \pi), \lim _{k \rightarrow \infty} \theta_{k}=\theta_{0} \in[0,2 \pi)$, $\left|g\left(z_{k}\right)\right|=M\left(r_{k}, g\right)$ and $k$ is sufficient large, we have

$$
\frac{f^{(j)}\left(z_{k}\right)}{f\left(z_{k}\right)}=\left(\frac{\nu_{g}\left(r_{k}\right)}{z_{k}}\right)^{j}(1+o(1)), \quad(j=0,1, \cdots, k)
$$

and

$$
\exp \left\{r_{k}^{\sigma-\varepsilon}\right\} \leq \nu_{g}\left(r_{k}\right) \leq \exp \left\{r_{k}^{\sigma+\varepsilon}\right\} .
$$

By the condition of Theorem 1.3 and Lemma 2.4, for any given $\varepsilon>0$, and the above $r_{k}\left(r_{k} \rightarrow \infty\right)$ such that

$$
\left|A_{j}\left(z_{k}\right)\right| \leq e^{\beta\left|z_{k}\right|^{\sigma+\varepsilon}}, \quad j=0,1, \cdots, k .
$$

Hence from the equation (1.1), we have

$$
\begin{aligned}
& \left(\frac{\nu_{g}\left(r_{k}\right)}{\left|z_{k}\right|}\right)^{n} \\
\leq & e^{r_{k}^{\sigma+\varepsilon}}\left(\frac{\nu_{g}\left(r_{k}\right)}{\left|z_{k}\right|}\right)^{n-1}|1+o(1)|+e^{r_{k}^{\sigma+\varepsilon}}\left(\frac{\nu_{g}\left(r_{k}\right)}{\left|z_{k}\right|}\right)^{n-2}|1+o(1)| \\
+ & \cdots+e^{r_{k}^{\sigma+\varepsilon}}\left(\frac{\nu_{g}\left(r_{k}\right)}{\left|z_{k}\right|}\right)|1+o(1)|+e^{r_{k}^{\sigma+\varepsilon}} .
\end{aligned}
$$

where the sequence $r_{k}\left(r_{k} \rightarrow \infty\right)$ satisfying $z_{k}=r_{k} e^{i \theta_{k}}, \theta_{k} \in[0,2 \pi), \lim _{k \rightarrow \infty} \theta_{k}=\theta_{0} \in$ $[0,2 \pi),\left|g\left(z_{k}\right)\right|=M\left(r_{k}, g\right)$. By (3.2)-(3.3), we get

$$
\limsup _{r \rightarrow \infty} \frac{\log \log \nu_{g}(r)}{\log r} \leq \sigma+\varepsilon .
$$

Since $\varepsilon$ is arbitrary, from Lemma 2.1, the above inequality and $\sigma_{2}(g) \leq \sigma$, we can obtain $\sigma_{2}(f) \leq \sigma$. Together with $\sigma_{2}(f) \geq \sigma$, we complete the proof of Theorem 1.3.

Proof of Theorem 1.4. Let $f \not \equiv 0$ be a meromorphic solution of infinite order of (1.1). We can get the equation of (3.1).

By Lemma 2.3, there exist a constant $c>0$ and a set $E_{1} \subset[0, \infty)$ having finite linear measure such that $|z|=r \notin E_{1}$ for all $z=r e^{i \theta}$. Then we have

$$
\left|\frac{f^{(j)}(z)}{f(z)}\right| \leq c[r T(2 r, f)]^{2 k}, \quad j=1, \ldots, k .
$$

By the hypotheses of Theorem 1.4, there exists a set $H$ with $\overline{\operatorname{dens}}\{|z|: z \in$ $H\}>0$ such that for all $z$ satisfying $z \in H$, we have

$$
\left|A_{0}(z)\right| \geq \exp _{p}\left\{\alpha|z|^{\mu}\right\}
$$




$$
\left|A_{j}(z)\right| \leq \exp _{p}\left\{\beta|z|^{\mu}\right\}, \quad j=1, \cdots, k-1, k,
$$

as $z \rightarrow \infty$.

Hence from (3.1), (3.4)-(3.6), it follows that for all $z$ satisfying $z \in H$ and $z \notin E_{1}$, we have

$$
\exp _{p}\left\{\alpha|z|^{\mu}\right\} \leq k \exp _{p}\left\{\beta|z|^{\mu}\right\} c[r T(2 r, f)]^{2 k}
$$

as $z \rightarrow \infty$. Thus, there exists a set $H_{1}=H \backslash E_{1}$ with $\overline{\operatorname{dens}}\left\{|z|: z \in H_{1}\right\}>0$ such that

$$
\exp _{p}\left\{(\alpha-\beta)|z|^{\mu}\right\} \leq k c[r T(2 r, f)]^{2 k}
$$

as $z \rightarrow \infty$. Therefore, by (3.8) and Definition 1, we obtain $\sigma_{p+1}(f) \geq \mu$.

Proof of Theorem 1.5. By Theorem 1.4, we have $\sigma_{p+1}(f) \geq \sigma-\varepsilon$, since $\varepsilon$ is arbitrary, we get $\sigma_{p+1}(f) \geq \sigma_{p}\left(A_{0}\right)=\sigma$. On the other hand, by Lemma 2.6, similar to the proof of Theorem 1.3. We can get

$$
\limsup _{r \rightarrow \infty} \frac{\log _{p+1} \nu_{g}(r)}{\log r} \leq \sigma+\varepsilon .
$$

Since $\varepsilon$ is arbitrary, by (3.9) and Lemma 2.1, we obtain $\sigma_{p+1}(f)=\sigma_{p+1}(g) \leq \sigma$. This and the fact that $\sigma_{p+1}(f) \geq \sigma$ yield $\sigma_{p+1}(f)=\sigma$.

Proof of Theorem 1.6. (i) First, we show that (1.8) can possess at most one exceptional solution $f_{0}$ satisfying $\sigma_{p+1}\left(f_{0}\right)<\sigma$ or $i\left(f_{0}\right)<p+1$. In fact, if $f^{*}$ is the another solution with $\sigma_{p+1}\left(f^{*}\right)<\sigma$ or $i\left(f^{*}\right)<p+1$, then $\sigma_{p+1}\left(f_{0}-f^{*}\right)<\sigma$ or $i\left(f_{0}-f^{*}\right)<p+1$. But $f_{0}-f^{*}$ is a solution of the corresponding homogeneous equation (1.1) to (1.8), this contradicts Theorem 1.5. We assume that $f$ is a solution with $\sigma_{p+1}(f) \geq \sigma$, and $f_{1}, f_{2}, \cdots, f_{k}$ is a solution base of the corresponding homogeneous equation (1.1). Then $f$ can be expressed in the form

$$
f(z)=B_{1}(z) f_{1}(z)+B_{2}(z) f_{2}(z)+\cdots+B_{k}(z) f_{k}(z),
$$

where $B_{1}(z), \cdots, B_{k}(z)$ are suitable meromorphic functions determined by

$$
\begin{gathered}
B_{1}^{\prime}(z) f_{1}(z)+B_{2}^{\prime}(z) f_{2}(z)+\cdots+B_{k}^{\prime}(z) f_{k}(z)=0, \\
B_{1}^{\prime}(z) f_{1}^{\prime}(z)+B_{2}^{\prime}(z) f_{2}^{\prime}(z)+\cdots+B_{k}^{\prime}(z) f_{k}^{\prime}(z)=0, \\
\vdots \\
B_{1}^{\prime}(z) f_{1}^{(k-1)}(z)+B_{2}^{\prime}(z) f_{2}^{(k-1)}(z)+\cdots+B_{k}^{\prime}(z) f_{k}^{(k-1)}(z)=F(z) .
\end{gathered}
$$

Since the Wronskian $W\left(f_{1}, f_{2}, \cdots, f_{k}\right)$ is a differential polynomials in $f_{1}, f_{2}, \cdots, f_{k}$ with constant coefficients, it is easy to deduce that $\sigma_{p+1}(W) \leq \sigma_{p+1}\left(f_{j}\right)=\sigma_{p}\left(A_{0}\right)=$ $\sigma$. From (3.11),

$$
B_{j}^{\prime}=F \cdot G_{j}\left(f_{1}, \cdots, f_{k}\right) \cdot W\left(f_{1}, \cdots, f_{k}\right)^{-1}, \quad j=1, \cdots, k,
$$


where $G_{j}\left(f_{1}, \cdots, f_{k}\right)$ are differential polynomials in $f_{1}, f_{2}, \cdots, f_{k}$ with constant coefficients, thus

$$
\sigma_{p+1}\left(G_{j}\right) \leq \sigma_{p+1}\left(f_{j}\right)=\sigma_{p}\left(A_{0}\right)=\sigma .
$$

Since $i(F)<p+1$ or $i(F)=p+1$ while $\sigma_{p+1}(F)<\sigma_{p}\left(A_{0}\right)$, by Lemma 2.7 and (3.13), for $j=1, \cdots, k$, we have

$$
\sigma_{p+1}\left(B_{j}\right)=\sigma_{p+1}\left(B_{j}^{\prime}\right) \leq \max \left\{\sigma_{p+1}(F), \sigma_{p}\left(A_{0}\right)\right\}=\sigma_{p}\left(A_{0}\right)=\sigma .
$$

Then from (3.10) and (3.14), we get

$$
\sigma_{p+1}(f) \leq \max \left\{\sigma_{p+1}\left(f_{j}\right), \sigma_{p+1}\left(B_{j}\right)\right\}=\sigma_{p}\left(A_{0}\right)=\sigma .
$$

This and the assumption $\sigma_{p+1}(f) \geq \sigma$ yield $\sigma_{p+1}(f)=\sigma$. If $f$ is a solution of the equation (1.8) satisfying $\sigma_{p+1}(f)=\sigma$, by Lemma 2.8, we have

$$
\bar{\lambda}_{p+1}(f)=\lambda_{p+1}(f)=\sigma_{p+1}(f)=\sigma .
$$

(ii) From the hypotheses of Theorem 1.6 and (3.10)-(3.15), we obtain

$$
\sigma_{q}(f) \leq \sigma_{q}(F)
$$

From (1.2), a simple consideration of order implies

$$
\sigma_{q}(f) \geq \sigma_{q}(F) .
$$

By this inequality and $(3.16), \sigma_{q}(f)=\sigma_{q}(F)$. This completes the proof of Theorem 1.6 .

Acknowledgment. The authors would like to thank the referee for many valuable suggestions and Professor Hongxun Yi for his encouragement and support.

\section{References}

[1] T. B. Cao, Z. X. Chen, X.M. Zheng and J. Tu, On the iterated order of meromorphic solutions of higher order linear differential equations, Ann. of Diff. Eqs., 21(2005), 111-122.

[2] Z. X. Chen, The growth of solutions of differential equation $f^{\prime \prime}+e^{-z} f^{\prime}+Q(z) f=0$, Science in China (series A), 31(2001), 775-784.

[3] Z. X. Chen, The zero, pole and order of meromorphic solutions of differential equations with meromorphic coefficients. Kodai Math. J., 19(1996), 341-354.

[4] Z. X. Chen, and C. C. Yang, Some further results on the zeros and growths of entire solutions of second order linear differential equations, Kodai Math. J., 22(1999), 273285. 
[5] M. Frei, Über die subnormalen Lösungen der Differentialgleichungen $w^{\prime \prime}+e^{-z} w^{\prime}+$ (konst.) $f=0$, Comment Math. Helv., 36(1961), 1-8.

[6] G. Gundersen, Estimates for the logarithmic derivative of a meromorphic function, Plus Similar Estimates, J. London Math. Soc., 37(1988), 88-104.

[7] G. Gundersen, Finite order solutions of second order linear differential equations, Tran. Amer. Math. Soc., 305(1988), 415-429.

[8] W. Hayman, Meromorphic Functions, Clarendon Press, Oxford, 1964.

[9] L. Kinnunen, Linear differential equations with solutions of finite iterated order, Southeast Asian Bull. Math., 22(1998), 385-405.

[10] I. Laine, Nevanlinna theory and complex differential equations, Walter de Gruyter, Berlin-New York, 1993.

[11] J. Wang and H. X. Yi, Fixed points and hyper order of differential polynomials generated by solutions of differential equation, Complex Var. Theory Appl., 48(2003), 83-94.

[12] L. Yang, Value Distribution Theory and New Research, Beijing, Science press, 1982.

[13] L. Z. Yang, Growth of linear differential equations and their applications, Israel J. Math, 147(2005), 359-372.

[14] H. X. Yi and C. C. Yang, The Uniqueness Theory of Meromorphic Functions, Science Press, Beijing, 1995. 